\title{
Nuclear lamins promote protrusion dynamics and collective, confined migration in vivo
}

\author{
Lauren Penfield ${ }^{\mathrm{a}}$ and Denise J. Montell ${ }^{\mathrm{a}, \mathrm{b}}$ \\ a Department of Molecular, Cellular, and Developmental Biology at University of California, Santa Barbara \\ ${ }^{\mathrm{b}}$ Correspondence: dmontell@ucsb.edu, Department of Molecular, Cellular, and Developmental Biology \\ University of California, Santa Barbara CA 93106
}

\begin{abstract}
Cells migrate collectively through confined environments during development and cancer metastasis. While the nucleus, a large and stiff organelle, impedes cell migration between non-deformable pillars in vitro, its function in vivo may vary depending on the microenvironment. Further, it is unknown how nuclei contribute to collective migration in vivo and whether nuclei in different positions within cell collectives experience different forces. Here, we use border cell migration in the fly ovary as an in vivo model to investigate the effects of confined, collective migration on nuclei and the contribution of nuclear lamins to migration. We found severe yet transient nuclear deformations occur, particularly in the leading cell, as border cells squeeze through tiny crevices between germline cells, termed nurse cells. Leading cells extend protrusions between nurse cells, which may pry open space to allow the cluster to advance. Here we report that the leading cell nuclei deformed as they moved into leading protrusions. Then as protrusions widened, the nucleus recovered a more circular shape. These data suggest that lead cell nuclei may help protrusions expand and thereby enlarge the migration path. To test how nuclei might promote or impede border cell migration, we investigated nuclear lamins, proteins that assemble into intermediate filaments and structurally support the nuclear envelope. Depletion of the Drosophila B-type lamin, Lam, from the outer, motile border cells, but not the inner, nonmotile polar cells, impeded border cell migration, whereas perturbations of the A-type lamin, LamC, did not. While wild type border cell clusters typically have one large leading protrusion as they delaminate from the anterior follicular epithelium, clusters depleted of B-type lamin had multiple, shortlived protrusions, resulting in unproductive cluster movement and failure to progress along the migration path. Further, border cell nuclei depleted of B-type lamins were small, formed blebs, and ruptured. Together, these data indicate that B-type lamin is requied for nuclear integrity, which in turn stabilizes the leading protrusion and promotes overall cluster polarization and collective movement through confined spaces.
\end{abstract}

Keywords: lamin, nucleus, collective cell migration, cell contact

\section{Introduction}

Collective cell migration is critical for development and wound healing, and promotes cancer metastases. One challenge cell clusters face when moving in vivo is that it is more difficult to move through small spaces such as dense extracellular matrices or between cells, for example during intravasation and extravasation ${ }^{1-3}$. How groups of cells physically squeeze through confined spaces and navigate complex tissue environments is not well understood.

Our lab and others have investigated confined, collective migration using the border cells in the Drosophila melanogaster ovary ${ }^{4,5}$. Drosophila ovaries contain strands of egg chambers progressing through 14 stages of development into the mature egg (Fig. 1A). Egg chambers consist of 16 germline cells that are surrounded by a layer of epithelial cells. Fifteen of the germline cells, termed nurse cells, supply nutrients and cytoplasm to the growing oocyte ${ }^{4,6}$. During stage 9 , two nonmotile cells called polar cells recruit 4-6 surrounding epithelial cells termed border cells (Fig. 1B) to surround and carry them. Together they move through crevices between the nurse cells until they arrive at the anterior oocyte border where they secrete a patterning signal and form a structure required for fertilization ${ }^{4,7}$. Thus, in the absence of border cell migration, females are sterile. Border cells are a well-established model for collective cell migration and have revealed key insights into how cells move in vivo ${ }^{4,5,7-}$
12. We recently showed that border cells always select the path with slightly larger spaces, which are located in the center of the egg chamber ${ }^{6}$. However, these extracellular openings are still much smaller than even a single cell, much less the whole cluster. Thus, how border cells move into these tiny spaces is not entirely clear. Border cells first extend protrusions, which function as sensory structures that probe the surroundings for chemoattractants and physical space $e^{6,7}$. A second and not mutually exclusive possibility is that protrusions function as a wedge to enlarge the openings between nurse cells.

The nucleus, typically the largest and stiffest organelle, has emerging mechanical roles in single cell migration ${ }^{13,14}$. In vitro, single cells have been studied migrating in dense collagen matrices or silicone microchannels that are narrower than the nucleus ${ }^{14,15}$. These studies show that nuclear deformation can be rate-limiting for cell movement and that nuclear stiffness impedes migration into confined spaces ${ }^{13-15}$. In contrast, emerging work suggests that nuclear stiffness enhances confined migration ${ }^{16,17}$. Researchers observing cells migrating in more deformable in vitro environments propose a nuclear piston model wherein stiffness facilitates the expansion of protrusions and forward movement in confined paths ${ }^{16,18}$. Other recent work finds that stretching of the nucleus in confined environments instigates a signaling cascade to activate myosin contractility and promote migration ${ }^{19,20}$. Thus, the role for nuclear mechanics may vary depending on context. Importantly, how 
Penfield et al., December, 2021- preprint copy - BioRxiv

nuclei contribute to collective, confined cell migration in vivo has not been thoroughly investigated. The role of the nucleus, if any, in border cell migration is unknown.

The mechanical properties of the nucleus depend on proteins that structure the nuclear envelope (NE), two lipid bilayers that define the nuclear compartment ${ }^{21}$. Lamins are proteins that form intermediate filament networks beneath the NE to provide structural support to the nucleus and organize chromatin and other NE proteins ${ }^{21,22}$. There are two types of lamins, A-type and B-type, which assemble into distinct filamentous networks with distinct properties. B-type lamins differ from A-type in that they retain a farnesyl post-translational modification that inserts into the inner nuclear membrane while this moiety is cleaved from A-type lamins ${ }^{22}$. Further, expression of A-type lamin, but not B-type, scales with tissue stiffness, and lamin A depletion decreases nuclear stiffness, so A-type lamins had been considered to be the main contributors to nuclear stiffness ${ }^{23-25}$. However, loss of B-type lamins can induce nuclear rupture and blebbing ${ }^{26,27}$, indicating that they also provide mechanical support. Further, overexpression of lamin B increases nuclear stiffness in human skin fibroblasts, HEK293 cells, and neuronal N2a cells ${ }^{28}$. Thus, B-type lamins have mechanical roles at the NE as well. While functions of A-type lamins have been investigated in confined migration ${ }^{14,15,29}$, roles for Btype lamins are far less clear.

Here, we report nuclear dynamics during collective border cell migration. We find that lead cell nuclei transiently deform more than follower cell or polar cell nuclei. Lead cell nuclei deformed as they moved into the neck of large, forward-directed protrusions, and nuclear translocation preceded protrusion widening. While perturbations of the Drosophila Atype lamin did not affect border cell migration, we found the Drosophila B-type lamin, Lam, promoted border cell migration and was required for maintaining a single leading protrusion. Together these data indicate nuclear structure promotes rather than hinders border cell migration. Specifically, the lead cell nucleus stabilizes the large, forwarddirected protrusion, which inhibits follower cell protrusions and promotes overall cluster polarity and directional migration. The lead cell also protects follower and polar cells from nuclear deformation.

\section{Results}

To study the role of nuclei in collective cell migration, we used border cell migration in the Drosophila egg chamber as a model (Fig. 1A-D). The cluster squeezes in between nurse cells to reach the oocyte by stage 10 . Typically, one outer border cell leads the cluster, extending and retracting protrusions as the cluster migrates (Fig. 1B), although cells can change position during migration with a follower cell overtaking the lead position $^{30}$.
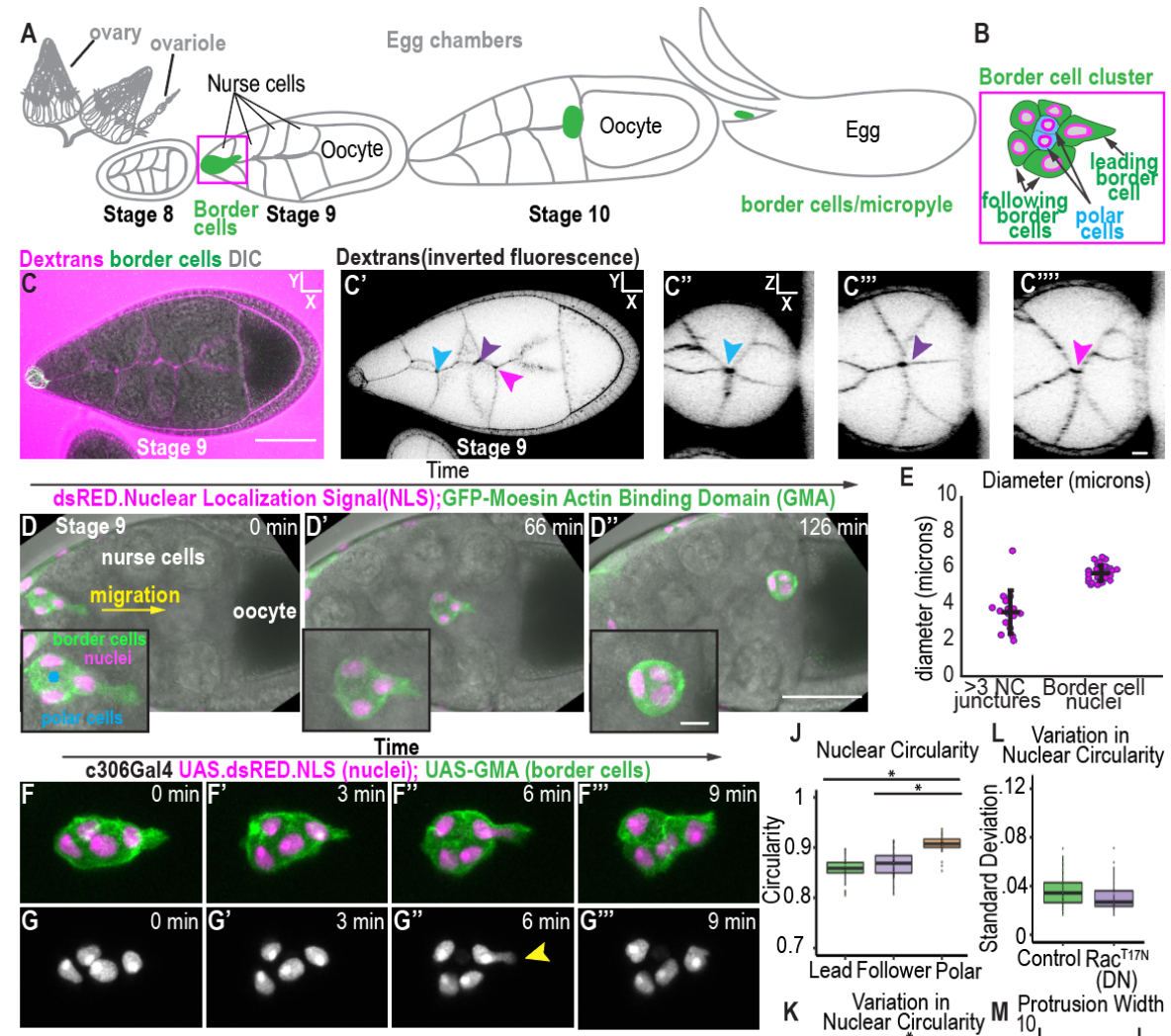

UpdGal4 UAS.dsRED.NLS (nuclei); UAS-GMA (polar cells)
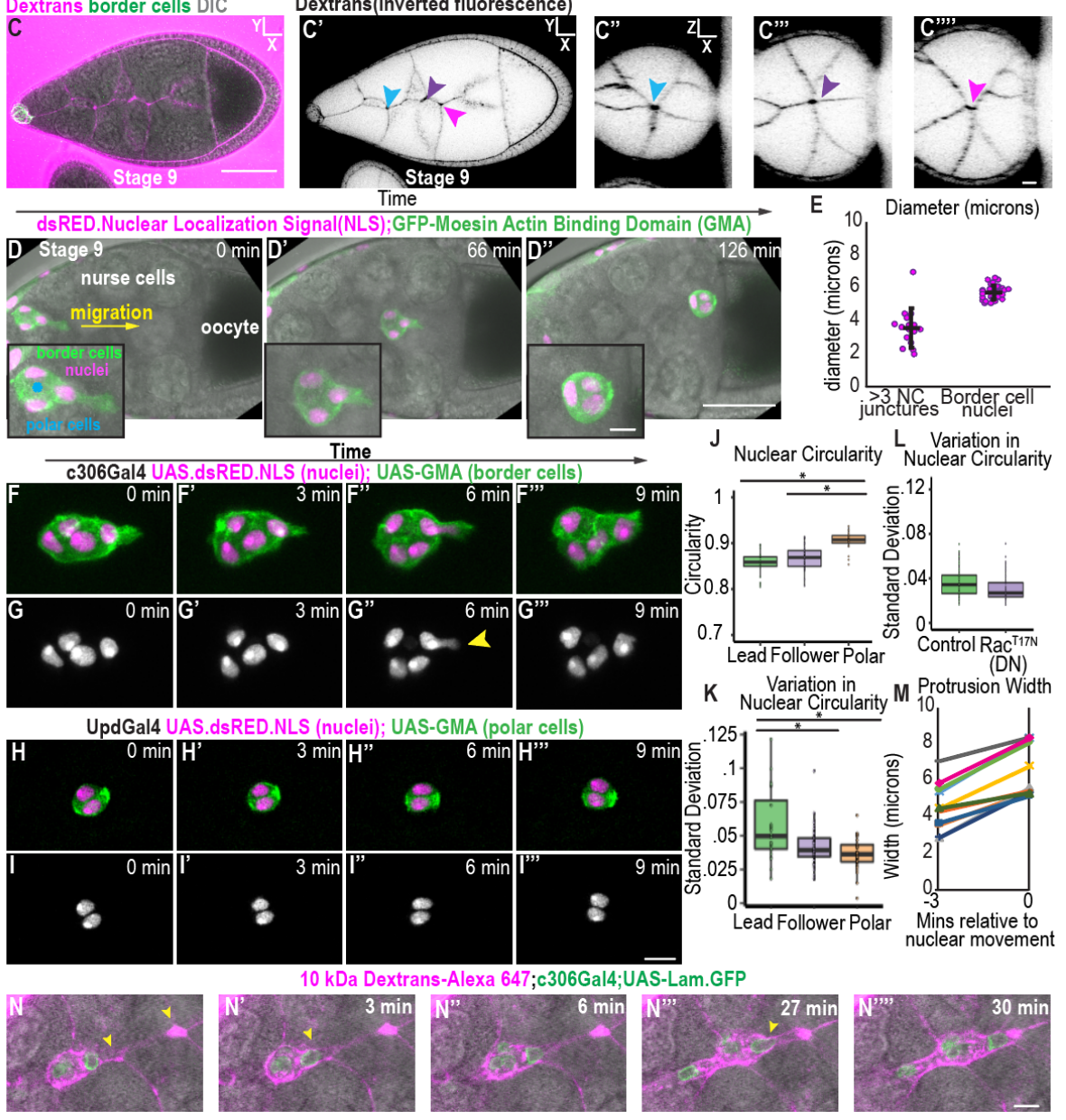

Fig. 1. Nuclei of leading border cells deform during migration into nurse cell junctures. $A$. Overview of the Drosophila ovary, ovariole, and egg chamber stages. B. Illustration of the border cells. Motile border cells (green) surround two nonmotile polar cells (blue). C. A stage 9 egg chamber incubated with fluorescent dextrans that fill extracellular spaces in magenta and expressing a slbo driven membrane marker, PLCdelta-EGFP, to mark border cells(green), DIC overlayed. Scale bar: $50 \mu \mathrm{m}$. C' inverted grayscale of the dextran signal; C" and C'". YZ view showing central and side paths. Scale bar: $10 \mu \mathrm{m}$. D. Images from timelapse with border cells (green), their nuclei (magenta), and the egg chamber (grey, DIC)), Scale bar: $50 \mu \mathrm{m}$. E. Plot showing individual and average +/standard deviation (SD) diameters of the $>3$ nurse cell junctures in the central path. F. Plot of individual and average +/- SD diameters of the border cell nuclei. G-J. Time lapse of nuclei in border or polar cells. Time is in minutes relative to the first frame shown. Scale bar: $10 \mu \mathrm{m}$. K. Plot of individual nuclear average circularity (dots) and median (center bar) average, nuclear circularity for the indicated conditions. L-M. Variation (standard deviation) of nuclear circularity over time for individual nuclei (dots) and the median variation in nuclear circularity (center bar) for the indicated conditions. * indicates $\mathrm{p}$ value less than .05 , see methods $\mathrm{N}$. Plot showing the maximum width of the protrusion before and after the maximum nuclear movement in the leading cell. Each line represents one leading cell. Scale bar: $10 \mu \mathrm{m}$.
There are two components to border cell path selection: border cells migrate posteriorly toward the oocyte and they always choose a central path, which contains slightly larger extracellular spaces than side paths. However these spaces are still smaller than a single border cell ${ }^{6}$. To 
Penfield et al., December, 2021 - preprint copy - BioRxiv
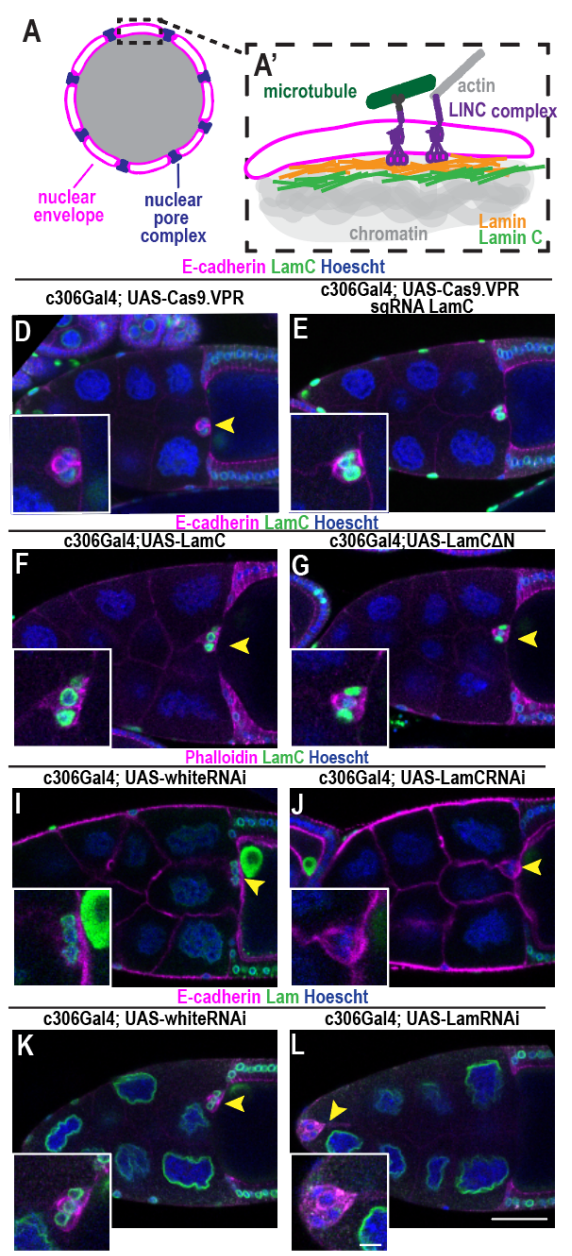

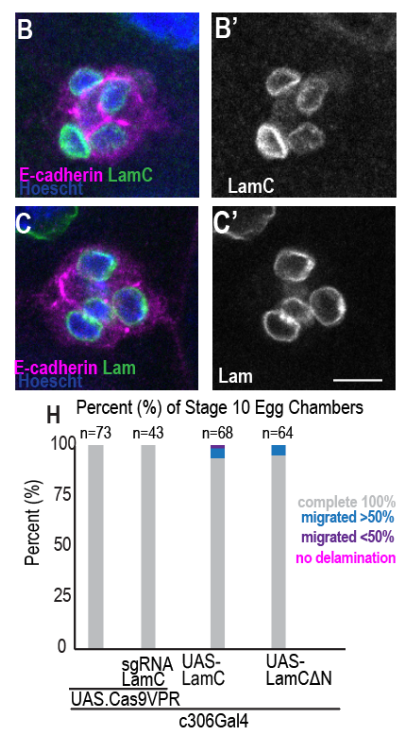

M Percent (\%) of Stage 10 Egg Chambers
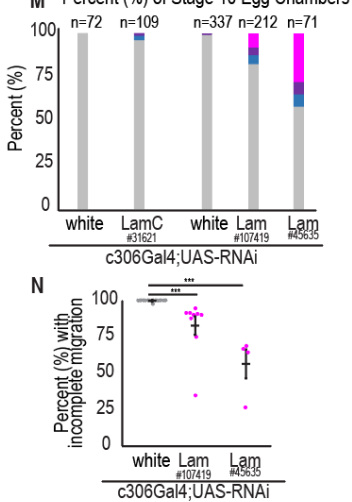

Fig. 2 B-type Lamins promote border cell migration. A. Schematic of the nuclear envelope. B-C. Images of fixed border cell clusters with the indicated markers and the staining for LamC (B') or Lam (C') in the border cell nuclei, Scale bar: $10 \mu \mathrm{m}$. D-G. Images of fixed stage 10 egg chambers for the indicated conditions, oocyte is always shown on the right and yellow arrowheads mark border cell cluster. Scale bar: $50 \mu \mathrm{m}$. H. Plots of the percent of stage 10 egg chambers with indicated migration phenotypes. $n=$ number of egg chambers counted. I-L. Images of fixed stage 10 egg chambers for the indicated conditions, yellow arrowheads mark the border cell cluster. Scale bar: $10 \mu \mathrm{m}$. M. Plots of the percent of stage 10 egg chambers with indicated migration phenotype. $n=$ number of egg chambers. N. Plot of individual experiments and average (+/-) of the percentages of egg chambers with incomplete migration at stage 10 for the indicated conditions. Asterisk $\left(^{*}\right)$ indicates $p$ value $<0.05$ (Mann Whitney $U$ test was performed)

investigate the effects of this confinement on border cell nuclei, we measured the diameter of the spaces between nurse cells along the central path compared to the diameters of border cell nuclei. We labeled extracellular spaces with fluorescent dextrans (Fig. 1C), and nuclei with dsRED-tagged with a nuclear localization signal (NLS) (Fig. 1D). The largest junctures between nurse cells averaged $<4$ microns, which was several microns smaller than the average minor axis of the nuclear diameter (Fig. 1E). Thus, nuclei are larger than the migration path, indicating that border cells must either make the path larger and/or undergo nuclear deformation to fit between nurse cells.

To assess if and when nuclei deform during border cell migration, we tracked the shape of nuclei with UAS-dsRED.NLS expressed in the outer border cells or in the inner polar cells by crossing UAS-fluorescent lines to Gal4s expressed in the border cells and/or polar cells (Fig. 1D, F-I). We also expressed an actin binding domain of moesin fused to GFP (UAS-GMA) to mark the border and/or polar cells (Fig 1. D, F-G). The nucleus in the leading cell underwent transient deformations and elongated along the axis of migration, however we never observed expulsion of dsRED.NLS from the nucleus, an indicator of NE rupture (Fig. 1F-G). The follower border cell nuclei were sometimes elongated but on average less dynamic than the border cells (Fig. 1F-G). In contrast, polar cell nuclei maintained a more stable shape during migration (Fig. 1H-I). These data indicate nuclear dynamics vary based on position and/or cell type within the cluster.

To quantify nuclear shape over time in border cells and polar cells, we measured the circularity of each nucleus at each time point of live imaging movies. Nuclear circularity values of 1 indicate a perfect circle while lower values indicate deviations from circular. Outer border cells have a lower nuclear circularity on average compared to inner, nonmotile polar cells (Fig. 1J). Further, the leading cells have the highest variation in nuclear circularity over time compared to following and polar cells (Fig. 1K). To determine if these transient changes in nuclear shape occurred due to migration between nurse cells, we compared nuclear circularity in border cells expressing a dominant negative form of Rac (RacN17). RacN17 expressing cells did not migrate or generate protrusions as previously reported ${ }^{12}$. The nuclei had significantly lower variation in nuclear shape compare to nuclei in control, migrating border cells (Fig 1L). Thus, outer border cell nuclei, particularly when leading the cluster, undergo transient shape changes as they migrate.

The leading cell nucleus often deformed while moving into the "neck" of the major forward-directed protrusion at the front of the cell (Fig. $1 \mathrm{~F}$ ') and expanded its diameter after moving past the protrusion neck (Fig. 1F','). To determine how intracellular nuclear movement affects protrusion size across samples, we measured the width before and after nuclear movement into the lead cell protrusion neck (Fig. 1M). The width of the protrusion consistently expanded after nuclear movement into the leading cell protrusions (Fig. 1M), suggesting nuclear movement causes the protrusions to expand.

To investigate how path size corresponded with nuclear deformation and movement, we expressed GFP::Lamin in the border cells to mark the nuclear envelope and used fluorescent dextran to fill extracellular spaces (Fig. 1N). The nucleus elongated along the axis of migration when the lead cell squeezed into smaller junctures between nurse cells, and recovered a more circular shape when the cell reached larger nurse cell junctures (Fig. 1N). These data indicate nuclei deform while moving into small junctures and undergo large movements and restore their circularity when reaching larger multicellular junctures.

In some in vitro experimental paradigms, nuclei impede migration when they have to deform to fit through a small opening ${ }^{14}$. However in other paradigms, nuclear stiffness can promote migration ${ }^{16,17}$. To test the functions of nuclei in border cell migration, we disrupted nuclear structure by altering the expression of lamins since they structurally support the NE and are mechanically coupled to the cytoskeleton through the Linker of Nucleoskeleton and Cytoskeleton (LINC) complex (Fig. $2 \mathrm{~A})^{22}$. The Drosophila genome encodes two lamins: an A-type lamin called LamC and a B-type lamin named $\mathrm{Lam}^{31,32}$. To test if they are expressed in border cells, we stained egg chambers for Lam and LamC. Both Lam and LamC are expressed in the border cells and localize to the nuclear periphery as expected (Fig 2B-C). 
Penfield et al., December, 2021- preprint copy - BioRxiv

A-type lamins are typically considered the major contributors to nuclear stiffness ${ }^{23,33}$. Using two different approaches (see methods for details), we over-expressed LamC using c306Gal4 (Fig. 2D-F), which is expressed in the border cells and polar cells (Fig. 1B). Although we detected increased expression of LamC compared to the control, we did not detect impairment of border cell migration at stage 10 (Fig. 2D-H). To disrupt LamC function, we overexpressed an N-terminal truncation (LamC $\Delta \mathrm{N})$, which was previously shown to decrease nuclear stiffness in Drosophila muscle nuclei ${ }^{34}$. Expression of LamC $\Delta \mathrm{N}$ causes LamC to localize in the nucleoplasm rather than at the nuclear rim in border cells (Fig. 2G). However, border cells completed migration by stage 10 with overexpression of the N-terminal deletion (Fig. 2G-H). As an alternative method to disrupt LamC function, we expressed an UAS-LamCRNAi line in the border and polar cells, which effectively depleted LamC compared to the control UAS-RNAi (Fig. 2I-J). However, Lam-C depleted egg chambers were able to reach the oocyte (Fig. 2I-J). Together these data show perturbations in LamC do not impede border cell migration.

B-type lamins also contribute to nuclear mechanics and maintenance of nuclear structure ${ }^{26-28,35,36}$. Thus, we also tested roles for Lam, the B-type lamin, in border cell migration. We found expression of 2 different UASRNAi lines targeting separate regions of the B-type lamin gene with c306Gal4 caused significant migration defects at stage 10 (Fig. 2K-N). The incomplete penetrance of the effect was due at least in part to incomplete knockdown. Thus, expression of Lam, the B-type lamin, promotes normal border cell migration and seems especially important for delamination.

To investigate how Lam depletion affects border cell dynamics, we performed live imaging to compare c306Gal;UAS-LamRNAi expressing border cells to the control border cells expressing c306Gal4;UASwRNAi (Fig. 3A-C). The control clusters extended one large, forwarddirected protrusion and traveled down the center of the egg chamber (Fig.
3A). While almost all control RNAi clusters delaminated during imaging, $43 \%$ of clusters expressing LamRNAi (\#45635) and 50\% of LamRNAi (\#107419)-expressing clusters failed to delaminate (Fig. 3D). Lamindepleted clusters extended protrusions in all of the clusters we observed, however protrusions were short lived compared to control clusters (Fig 3B-C, E). Frequently, the lead cell nucleus did not move into the protrusion (Fig. 3B), however when Lam-depleted nuclei attempted to move forward (Fig. 3C'), nuclei often quickly withdrew from the protrusion, which was followed by narrowing and retraction of the protrusion (Fig 3C',-C',',). In addition, ectopic protrusions commonly extended to the side of the egg chamber (33\% of \#107419 LamRNAi and $75 \%$ \#45635) (Fig 3B, F). Possibly as a consequence, clusters moved between the follicle cells and germ cells instead of taking their normal central path (Fig. 3B). Together these data indicate Lam is required to maintain the leading protrusion, prevent ectopic protrusions, and move through the center of the egg chamber.

Because Lam depletion affected the dynamics of the protrusions, which are only produced by outer, border cells, we predicted that Lam would be required in the border cells rather than the polar cells for migration. To test this hypothesis, we depleted Lam specifically in the border cells with fruitlessGal4 or in the inner polar cells with Unpaired(Upd)Gal4 (Fig. 3G-H). FruitlessGal4 caused migration defects when combined with either LamRNAi lines whereas UpdGal4 did not (Fig. 3I). Together these findings indicate Lam expression in the nuclei of the outer border cells helps maintain a single leading protrusion, cluster polarity, and directional migration.

In Lam-depleted border cell clusters that were able to migrate, we noticed even more severe nuclear deformations in the leading cells than wild type. While control nuclei elongated as they moved into protrusion necks (Fig. 4A), nuclei in leading cells depleted of Lam formed blebs in the opposite direction of the protrusion (Fig. 3C', 4B), indicating that Lam normally opposes a rearward force. Nuclear blebs have been observed to
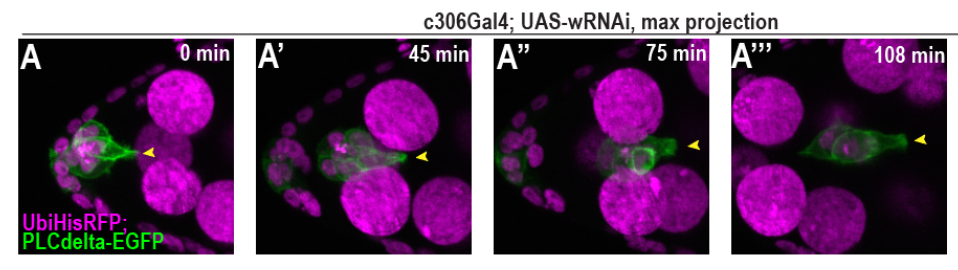

c306Gal4; UAS-LamRNAi, max projection
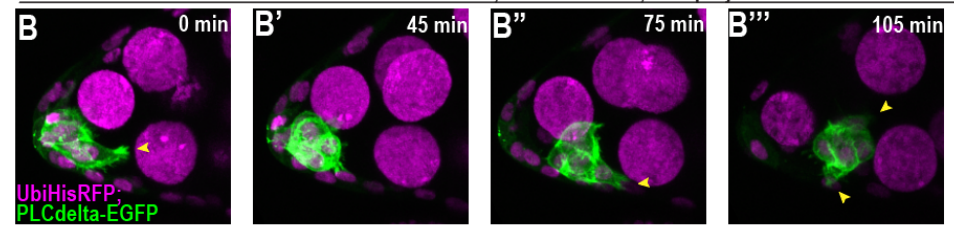

c306Gal4; UAS-LamRNAi, single z-slice
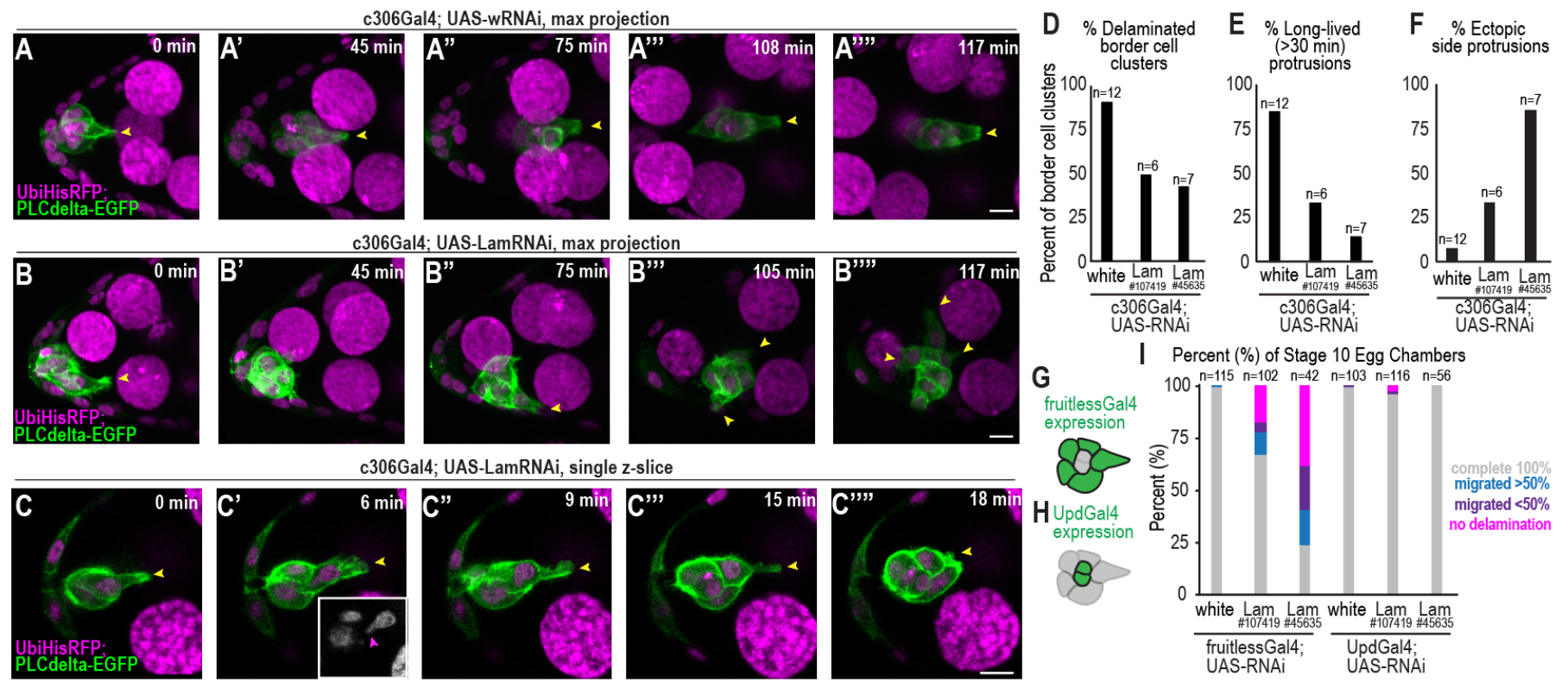

Fig. 3. Lam is required in the outer border cells to coordinate protrusion dynamics. A-C. Max projections (A-B) or single Z-slice (C) images from time lapses of stage 9 egg chambers expressing Ubiquitin-driven histone tagged with RFP (UbiHisRFP0 and a slbo(border cell gene)-driven membrane marker, PLC-delta-GFP) Yellow arrowheads mark protrusions and magenta arrowhead marks a nuclear bleb Scale bar: $10 \mu \mathrm{m}$. D-F. Plots quantifying phenotypes from live imaging of border cell clusters in control RNAi or Lam RNAi conditions. G-H. Schematics showing the expression for the indicated Gal4s. I. Plot of the percent of stage 10 egg chambers with indicated migration phenotypes, $n=$ number of egg chambers. 
Penfield et al., December, 2021 - preprint copy - BioRxiv
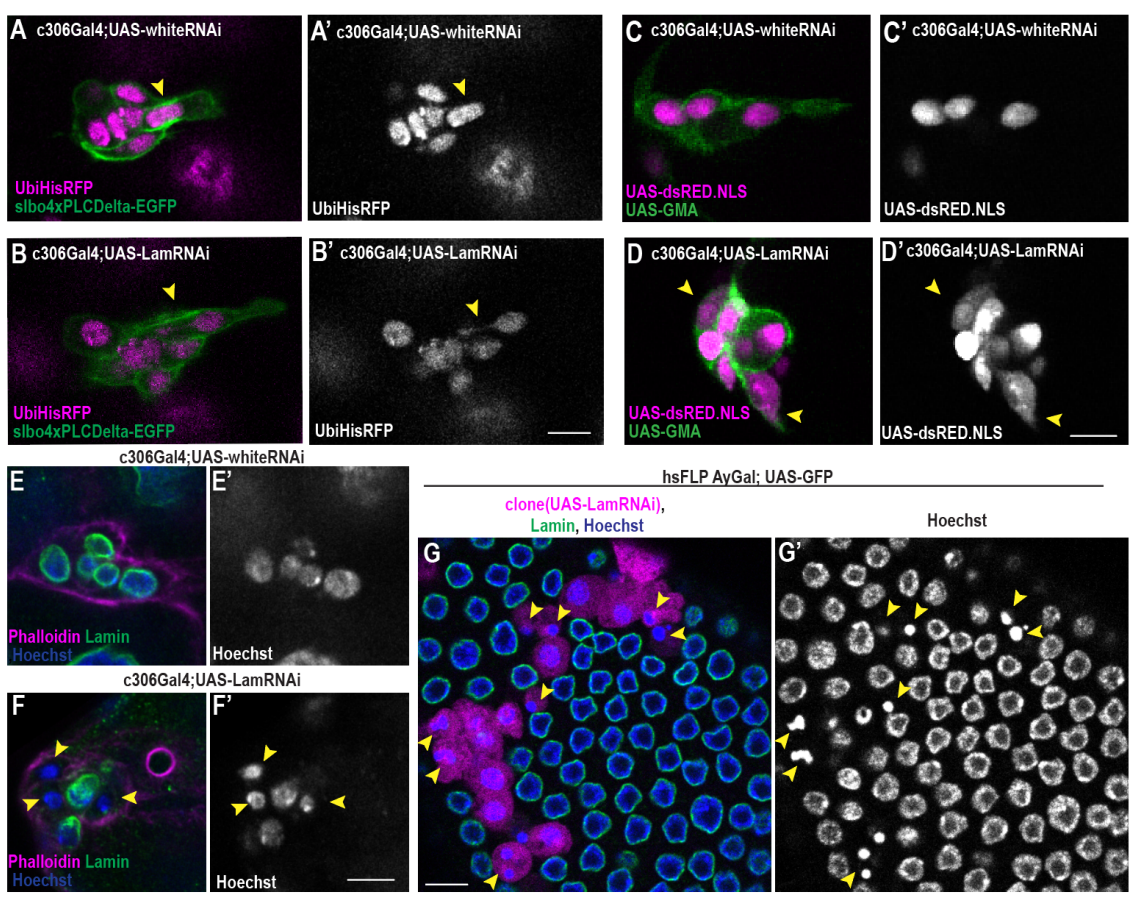

SFLP AYGal; UAS-GFP

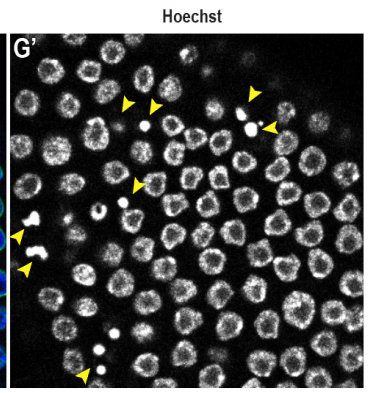

Fig. 4 Lamins maintain nuclear structure and size in border cells and nonmigratory follicle cells. A-B. Select images from timelaspe series for the indicated conditions and fluorescent reporters. Arrowhead marks nuclear bleb in B and B' Scale Bar: $10 \mu \mathrm{m}$. C-D. Select images from timelapse series for the indicated markers. Arrowheads mark expulsion of the dsRED.NLS into cells, an indicator of nuclear rupture. E-F. Representative images from fixed imaging of control (white) or Lam-depleted border cell clusters. Yellow arrowheads point to smaller nuclei Scale Bar: $10 \mu \mathrm{m}$. G. Representative image from hsFLP AyGal4; UAS-GFP UAS-LamRNAi egg chambers. Yellow arrowheads point to smaller nuclei in LamRNAi clones. Scale Bar: $10 \mu \mathrm{m}$.

precede nuclear rupture in other lamin-depleted cells ${ }^{26,37}$. To assess how Lam affects the nuclear permeability barrier in the border cells, we monitored nuclei with dsRED.NLS which localizes to the nucleus when the nuclear barrier is intact. In border cells expressing a control UASRNAi, we never observed nuclear rupture ( $n=4$, Fig. $4 C$ ), whereas the dsRED.NLS diffused into the cytoplasm in $50 \%$ of Lam-depleted border cells ( $n=8$, Fig. 4D). Leakage of dsRED.NLS into the cytoplasm was observed in border cells with protrusions as well as other Lam-depleted cells in the egg chamber (Fig. 4D), consistent with reports that nuclei of lamin depleted cells rupture spontaneously in culture ${ }^{26,38,39}$.

While Lam-depleted nuclei appeared to have a similar shape in following border cells compared to controls (Fig. 4A-B), analysis of fixed border cell clusters that failed to delaminate revealed that a subset of lamindepleted nuclei were smaller than nuclei in control clusters (Fig. 4E-F); the smaller nuclei tended to have a more effective depletion of Lam than larger nuclei as observed by immunostaining for Lam (Fig. 4F). To directly compare nuclear size in the same egg chamber of cells with or without Lam, we performed FlpOUT experiments to generate mosaic egg chambers with some cells expressing Lamin-RNAi. Nuclei were smaller in Lam-depleted follicle cells from which the border cells derive compared to clones that did not express the LamRNAi (Fig. 4G). Thus, Lam is required to maintain both nuclear size and the stability of the nuclear barrier, suggesting maintaining nuclear structure is important for border cell migration.

\section{Discussion}

The functions of nuclei in confined migration in vivo are poorly understood. Further, functions of nuclei during collective migration have not been thoroughly investigated. Here, we report the dynamics of nuclei during border cell migration and reveal a role for nuclear structure in this collective, confined, in vivo cell migration. We show that the following cells and inner polar cells undergo less deformation than the leading cell, suggesting that one advantage of collective movement is follower cell nuclei are protected from deformations by the lead cell. Since severe deformations can result in nuclear rupture and DNA damage ${ }^{38-40}$, such protection could be physiologically significant for cells moving collectively during wound healing and tumor metastasis. We find that nuclear deformation occurs as the nucleus moves into the narrow protrusion neck. Nuclear movement into and then past the protrusion neck precedes and correlates with widening of the leading protrusion, suggesting that the nucleus may function as a "plug" to prevent protrusion collapse and facilitate its expansion (Fig. 5A). Since depletion of nuclear lamins disrupts border cell migration, the data suggest that nuclear structure promotes rather than impedes this confined in vivo migration.

We previously showed that $>90 \%$ of forward-directed protrusions eventually retract, and that the speed of migration is relatively constant regardless of whether the lead cell is protruding or retracting ${ }^{11}$. We suggested that protrusions are sensory structures that probe the microenvironment for chemoattractants and for physical space ${ }^{6,11}$. The new data suggest an additional function for the lead cell protrusion - physical enlargement of the available space in front of the cluster to facilitate movement. The data suggest that growth and enlargement of the lead cell protrusion, which is

\section{Time}

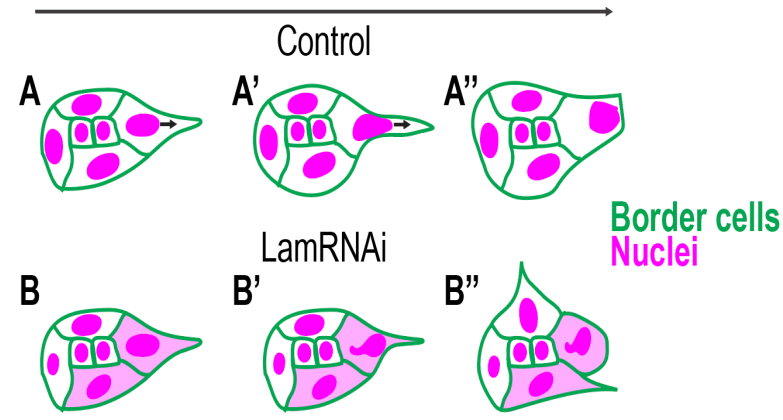

Fig. 5 Model for the role of the nucleus in border cell migration $A$. Control border cell cluster model. One border cell extends a protrusion into the central path followed by nuclear elongation and movement to plug the protrusion neck and stabilize the protrusion ( $\left.A^{\prime}\right)$. Nuclear movement through the protrusion neck further widens the protrusion ( $\left.\mathrm{A}^{\prime \prime}\right)$. B. Model of LamRNAi phenotype. Protrusions extend into the central path when Lam is depleted, but the nucleus is unable to stabilize the protrusion neck and is pushed backward (B'). Without a stable leading protrusion, other protrusions then extend and retract, leading to uncoordinated movement (B"). 
Penfield et al., December, 2021- preprint copy - BioRxiv

facilitated by the movement of the stiff nucleus into the base of the protrusion, is essential for cluster polarization and movement between nurse cells.

Depletion of Lam results in border cells with multiple, short-lived protrusions (Fig. 3), indicating that lamin at the nuclear envelope promotes enlargement and stabilization of the lead cell protrusion. Our previous work shows that mechanical feedback from the dominant lead protrusion inhibits side and rear protrusion ${ }^{8}$. Thus, without maintenance of a single protrusion towards the center of the egg chamber in Lamdepleted cells, overall cluster front/back polarization fails to stabilize, side protrusions extend, and clusters either fail to migrate or take an abnormal trajectory (Fig. 5B). Based on our data, we predict that B-type lamins maintain nuclear size and structure, which are important to counteract rearward forces at protrusion necks, to prevent protrusion collapse, and to facilitate protrusion growth. While we have not ruled out the possibility that lamin is also required to regulate gene expression, which is another function of the nuclear lamina ${ }^{22}$, border cell fate and differentiation and their ability to generate dynamic protrusions is unaffected, suggesting a mechanical function is most likely.

We previously described the extracellular space in center of the egg chamber as the energetically most favorable path due to the presence of slightly larger spaces at multiple nurse cell junctures compared to interfaces between two nurse cells ${ }^{6}$. However this effect is strongest in the middle half of the egg chamber where extracellular spaces are largest ${ }^{6}$, and border cells are more susceptible to migrating off the central path at the very beginning and the very end of migration, where the topological cue is weakest. This may explain why the lamin-depleted clusters, which struggle to develop a major protrusion and detach from the anterior end, sometimes move between follicle cells and nurse cells. This finding indicates that the central path is only more favorable when nuclear stiffness is in the normal range and can stabilize the leading protrusion. For Lam RNAi-expressing clusters, the inability to enlarge the lead protrusion eliminates the advantage of the central path.

Intriguingly, levels of LamC, the Drosophila A-type lamin, do not affect migration of border cells while levels of B-type lamin do (Fig. 2). In contrast, work almost exclusively performed on single cells in vitro, found roles for A-type lamins in confined, cell migration ${ }^{14-16,29}$. There are several possible explanations for this result. One possibility is that LamC is not as highly expressed in the border cells, so B-type lamins contribute more to nuclear mechanics in border cells. Alternatively, Btype lamins might have a different mechanical function than A-type lamins in the border cells. Prior work has indicated that B-type lamins contribute more to nuclear elasticity than nuclear viscosity ${ }^{24}$. B-type lamins have been proposed to act as the walls of an elastic balloon while A-type lamins act as a viscous fluid to resist deformation ${ }^{24}$. In border cells, B-type lamins may maintain elasticity to allow nuclei to recover after being stretched to move into and widen protrusions necks. Alternatively, since we see nuclei are smaller upon Lam depletion, Btype lamins may be required to generate nuclei that are large enough to "plug" protrusions and prevent their collapse. Future work is required to fully dissect how B-type lamins versus A-type lamins contribute to nuclear mechanics in border cells and other migratory cells.

\section{Materials and Methods}

\section{Drosophila genetics}

Drosophila stocks used in this study are described in Table 1. Crosses were established with 5-7 virgin females and crossed to 2-3 males to combine Gal4s with different UAS-lines. Drosophila stocks were maintained at $25{ }^{\circ} \mathrm{C}$ degrees for genetic crosses and flies were flipped into new vials every 2-3 three days. Prior to dissection, flies were fattened at $29^{\circ} \mathrm{C}$ for 1 day.

To overexpress LamC, we expressed a UAS-LamC line ${ }^{34,41}$ in the border cells with c306Gal4. Additionally, we used another previously published method $^{42}$ to overexpress LamC by co-expressing a catalytically inactive form of Cas 9 that was tethered to transcriptional machinery (UASCas9.VPR), along with a sgRNA targeting LamC using c306Gal4.

Table 1. Drosophila Stocks.

\begin{tabular}{|c|c|}
\hline Stock description and genotype & Source \\
\hline c306-Gal4 & Lab stock \\
\hline Fruitless-Gal4 & Lab stock \\
\hline Upd-Gal4 & Lab Stock \\
\hline $\begin{array}{l}\text { hsFLP, AyGal4; UAS-GFP } \\
\mathrm{y}[1] \mathrm{w}[*] ; \mathrm{P}\{\text { ry }[+\mathrm{t} 7.2]=\mathrm{hsFLP}\} 12, \\
\mathrm{P}\{\mathrm{w}[+\mathrm{mC}]=\text { AyGAL4 }\} 25 \\
\mathrm{P}\{\mathrm{w}[+\mathrm{mC}]=\mathrm{UAS}- \\
\text { GFP.S65T }\} \text { Myo31DF[T2] }\end{array}$ & $\begin{array}{l}\text { Lab Stock } \\
\text { BDSC \#4411 and \#1929 }\end{array}$ \\
\hline UAS-LamRNAi & VDRC, \#107419 \\
\hline UAS-LamRNAi & VDRC, $\# 45635$ \\
\hline UAS-whiteRNAi/CyO & Lab stock \\
\hline $\begin{array}{l}\text { UAS-Cas9.VPR } \\
\mathrm{y}[1] \mathrm{w}[*] ; \mathrm{Kr}[\mathrm{If}-1] / \mathrm{CyO} ; \mathrm{P}\{\mathrm{y}[+\mathrm{t} 7.7] \\
\text { w[+mC }]=\text { UAS- } \\
\text { 3xFLAG.dCas9.VPR }\} \text { attP2 }\end{array}$ & BDSC, \# 66562 \\
\hline $\begin{array}{l}\text { LamC guideRNA } \\
\mathrm{y}[1] \mathrm{sc}\left[{ }^{*}\right] \mathrm{v}[1] \text { sev[21]; P }\{\mathrm{y}[+\mathrm{t} 7.7] \\
\mathrm{v}[+\mathrm{t} 1.8]=\text { TOE.GS01246 }\} \text { attP40 }\end{array}$ & BDSC, $\# 79670$ \\
\hline $\begin{array}{l}\text { UAS-LamCRNAi }(\mathrm{y}[1] \mathrm{v}[1] ; \\
\mathrm{P}\{\mathrm{y}[+\mathrm{t} 7.7] \\
\mathrm{v}[+\mathrm{t} 1.8]=\text { TRiP.JF01406 }\} \mathrm{attP} 2\end{array}$ & BDSC, \#31621 \\
\hline $\begin{array}{l}\mathrm{y}[1] \mathrm{sc}[*] \mathrm{v}[1] \operatorname{sev}[21] ; \mathrm{P}\{\mathrm{y}[+\mathrm{t} 7.7] \\
\mathrm{v}[+\mathrm{t} 1.8]=\text { TOE.GS01246 }\} \text { attP40 }\end{array}$ & BDSC, 79760 \\
\hline $\begin{array}{l}\mathrm{y}[1] \mathrm{w}[*] ; \mathrm{P}\{\mathrm{y}[+\mathrm{t} 7.7] \\
\mathrm{w}[+\mathrm{mC}]=\mathrm{UAS}- \\
\text { 3xFLAG.dCas9.VPR }\} \text { attP40 }\end{array}$ & BDSC, 66561 \\
\hline UAS-LamC & Wallrath Lab \\
\hline UAS-LamCdeltaN & Wallrath Lab \\
\hline $\begin{array}{l}P[U A S \text {-dsRed.NLS]; P[UAS-moesin- } \\
\text { GFP], }\end{array}$ & Lab stock \\
\hline $\begin{array}{l}\mathrm{y}[1] \mathrm{w}[*] ; \mathrm{P}\{\mathrm{w}[+\mathrm{mC}]=\mathrm{UAS}- \\
\text { Lam.GFP }\} 3-3\end{array}$ & BDSC, \#7376 \\
\hline $\begin{array}{l}\text { w; ubi-HisRFP, slbo- } \\
\text { 4xPLCdeltaEGFP }\end{array}$ & Lab Stock \\
\hline
\end{tabular}


Penfield et al., December, 2021 - preprint copy - BioRxiv

\section{Immunostaining and Fixed Imaging}

Ovaries were dissected in live imaging media (Schneider's media (Thermo Fisher Scientific) +20\% Fetal Bovine Serum) and fixed in 4\% paraformaldehyde in 1X Phosphate-buffered saline (PBS) for 15 minutes. Ovarioles were washed with $1 \mathrm{X}$ PBS $+0.4 \%$ triton (PBST) 4 times total for 10 minutes each time and incubated in primary antibodies overnight at $4{ }^{\circ} \mathrm{C}$. Antibodies used include: Lam antibody (DHSB ADL84.12) E-cadherin antibody (DHSB DCAD2), LamC (LC28.26). The following day, ovaries were washed with 1X PBST followed by 3 additional washes $1 \mathrm{X}$ PBST for 10 minutes. Then, ovaries were incubated with secondary antibodies (Goat anti-Rat IgG $(\mathrm{H}+\mathrm{L})$ Cross-Adsorbed Secondary Antibody, Alexa Fluor 647 and 568, Invitrogen, Goat anti-Mouse IgG $(\mathrm{H}+\mathrm{L})$ Cross-Adsorbed Secondary Antibody, Alexa Fluor 488 and 568, Invitrogen), phalloidin, and Hoechst for 1 hour. Ovaries were washed with 1XPBST followed by 3 additional washes $1 \mathrm{X}$ PBST for 10 minutes. After the last wash, ovaries are suspended in vectashield (H-1000, Vector Laboratories) and incubated overnight at $4{ }^{\circ} \mathrm{C}$ before mounting onto slides.

Slides were imaged on a Zeiss LSM780 confocal microscope with a $40 \times$ 1.1 N.A. $0.62 \mathrm{~mm}$ long working distance water objective.

\section{Live Imaging}

Strands of ovarioles were dissected from ovaries in live imaging media and resuspended in live imaging media with insulin and $1 \%$ agarose and immediately mounted for imaging. Stage 9 egg chambers were imaged on an inverted Zeiss LSM800 confocal microscope fitted with a PlanApochromat 40X, 1.2 NA multi-immersion objective with the collar set for water immersion at 3-minute time intervals.

For imaging with dextrans to mark extracellular spaces, fluorescent dextrans were added to the media prior to mounting $(100 \mu \mathrm{g} / \mathrm{ml}$; Dextran Alexa 647 10,000 MW; Thermo Fisher Scientific; D22914).

\section{Image analysis and Statistical Analysis}

To measure nuclear circularity from time-lapse series, the central z-slice of the channel with UAS.dsRED.NLS in each nucleus was cropped from each time point to prevent overlap of nuclei during semiautomated analysis. Nuclei were segmented into binary images using a code generated in Matlab and shape parameters were measured over time using Image $\mathrm{J}$ measurements. All time points were included unless the nucleus went out of focus during the imaging session. The average and standard deviation of nuclear circularity was measured for each nucleus. Statistical testing was performed in $\mathrm{R}$ using Mann Whitney $\mathrm{U}$ test for migration defects or an ANOVA blocked by movie variation with Tukey post-hoc analysis for nuclear shape analysis.

To measure protrusion width, lines were drawn across the protrusion at the widest position and measured in Image $\mathbf{J}$ over time. To measure intracellular nuclear movement in the leading cell, a line was drawn from the exterior of the nucleus to the center of the tip of the protrusion in Image J.

To measure border cell migration defects, all stage 10 egg chambers were identified and the border cell cluster was detected with E-cadherin or phalloidin staining. All egg chambers were scored except with those with physical damage from dissection. Then, the position of the cluster was recorded for each stage egg chamber. RNAi experiments were performed with at least 3 independent experiments and genetic crosses, and with the control (white) RNAi line in parallel. Overexpression experiments were performed with at least 2 independent experiments.

\section{End Matter}

\section{Author Contributions and Notes}

L.P. and D.J.M. designed research, L.P. performed research, L.P. analyzed data; and L.P and D.J.M wrote the paper.

The authors declare no conflict of interest.

\section{Acknowledgments}

This work was supported by NIH grant R01GM073164 to D.J.M. We would like to thank the Bloomington Drosophila Stock Center, the Vienna Drosophila Resource Center (VDRC), and Lori Wallrath for the Drosophila stocks. We would like to thank Carinna Tran and Jim Mondo for their technical assistance.

\section{References}

1. $1 . \quad$ Stoletov, K. et al. Visualizing extravasation dynamics of metastatic tumor cells. J. Cell Sci. 123, 2332 LP-2341 (2010).

2. Wyckoff, J. B., Jones, J. G., Condeelis, J. S. \& Segall, J. E. A critical step in metastasis: in vivo analysis of intravasation at the primary tumor. Cancer Res. 60, 2504-11 (2000).

3. Reymond, N., d'Água, B. B. \& Ridley, A. J. Crossing the endothelial barrier during metastasis. Nat. Rev. Cancer 13, 858870 (2013).

4. Montell, D. J. Border-cell migration: the race is on. Nat. Rev. Mol. Cell Biol. 4, 13-24 (2003).

5. Mishra, A. K., Campanale, J. P., Mondo, J. A. \& Montell, D. J. Cell interactions in collective cell migration. Development 146, dev172056 (2019).

6. Dai, W. et al. Tissue topography steers migrating \&lt;em\&gt;Drosophila\&lt;/em\&gt; border cells. Science (80-. ). 370, 987 LP-990 (2020).

7. Montell, D. J., Yoon, W. H. \& Starz-Gaiano, M. Group choreography: mechanisms orchestrating the collective movement of border cells. Nat. Rev. Mol. Cell Biol. 13, 631-645 (2012).

8. Cai, D. et al. Mechanical Feedback through E-Cadherin Promotes Direction Sensing during Collective Cell Migration. Cell 157, 1146-1159 (2014).

9. Duchek, P., Somogyi, K., Jékely, G., Beccari, S. \& Rørth, P. Guidance of Cell Migration by the Drosophila PDGF/VEGF Receptor. Cell 107, 17-26 (2001).

10. Wang, X., He, L., Wu, Y. I., Hahn, K. M. \& Montell, D. J. Lightmediated activation reveals a key role for Rac in collective guidance of cell movement in vivo. Nat. Cell Biol. 12, 591-597 (2010).

11. Mishra, A. K., Mondo, J. A., Campanale, J. P. \& Montell, D. J. Coordination of protrusion dynamics within and between collectively migrating border cells by myosin II. Mol. Biol. Cell $\mathbf{3 0}$, 2490-2502 (2019)

12. Murphy, A. M. \& Montell, D. J. Cell type-specific roles for Cdc42, Rac, and RhoL in Drosophila oogenesis. J. Cell Biol. 133, 617630 (1996).

13. Calero-Cuenca, F. J., Janota, C. S. \& Gomes, E. R. Dealing with the nucleus during cell migration. Curr. Opin. Cell Biol. 50, 35-41 (2018).

14. Davidson, P. M., Denais, C., Bakshi, M. C. \& Lammerding, J. Nuclear Deformability Constitutes a Rate-Limiting Step During Cell Migration in 3-D Environments. Cell. Mol. Bioeng. 7, 293-306 (2014).

15. Harada, T. et al. Nuclear lamin stiffness is a barrier to $3 D$ migration, but softness can limit survival. J. Cell Biol. 204, 669- 
Penfield et al., December, 2021- preprint copy - BioRxiv

682 (2014).

16. Lee, $\mathrm{H}$. et al. The nuclear piston activates mechanosensitive ion channels to generate cell migration paths in confining microenvironments. Sci. Adv. 7, eabd4058 (2021).

17. Krause, M. et al. Cell migration through three-dimensional confining pores: Speed accelerations by deformation and recoil of the nucleus. Philos. Trans. R. Soc. B Biol. Sci. (2019). doi:10.1098/rstb.2018.0225

18. Petrie, R. J., Koo, H. \& Yamada, K. M. Generation of compartmentalized pressure by a nuclear piston governs cell motility in a 3D matrix. Science (80-. ). 345, 1062 LP-1065 (2014).

19. Lomakin, A. J. et al. The nucleus acts as a ruler tailoring cell responses to spatial constraints. Science (80-. ). 370, eaba2894 (2020).

20. Venturini, V. et al. The nucleus measures shape changes for cellular proprioception to control dynamic cell behavior. Science (80-. ). 370, eaba2644 (2020).

21. Hetzer, M. W. The nuclear envelope. Cold Spring Harbor perspectives in biology 2, a000539 (2010).

22. Davidson, P. M. \& Lammerding, J. Broken nuclei - lamins, nuclear mechanics, and disease. Trends Cell Biol. 24, 247-256 (2014).

23. Lammerding, J. et al. Lamin A/C deficiency causes defective nuclear mechanics and mechanotransduction. J. Clin. Invest. 113, 370-378 (2004).

24. Swift, J. et al. Nuclear Lamin-A Scales with Tissue Stiffness and Enhances Matrix-Directed Differentiation. Science (80-. ). 341, 1240104-1240104 (2013).

25. Lammerding, J. et al. Lamins a and $C$ but not lamin $B 1$ regulate nuclear mechanics. J. Biol. Chem. 281, 25768-25780 (2006).

26. Vargas, J. D., Hatch, E. M., Anderson, D. J. \& Hetzer, M. W. Transient nuclear envelope rupturing during interphase in human cancer cells. Nucleus 3, 88-100 (2012).

27. Hatch, E. M. \& Hetzer, M. W. Nuclear envelope rupture is induced by actin-based nucleus confinement. J. Cell Biol. 215, (2016).

28. Ferrera, D. et al. Lamin B1 overexpression increases nuclear rigidity in autosomal dominant leukodystrophy fibroblasts. FASEB J. 28, 3906-3918 (2014).

29. Rowat, A. C. et al. Nuclear envelope composition determines the ability of neutrophil-type cells to passage through micron-scale constrictions. J. Biol. Chem. (2013). doi:10.1074/jbc.M112.441535

30. Prasad, M. \& Montell, D. J. Cellular and Molecular Mechanisms of Border Cell Migration Analyzed Using Time-Lapse Live-Cell Imaging. Dev. Cell 12, 997-1005 (2007).

31. Lenz-Böhme, B. et al. Insertional mutation of the Drosophila nuclear lamin Dm0 gene results in defective nuclear envelopes, clustering of nuclear pore complexes, and accumulation of annulate lamellae. J. Cell Biol. 137, 1001-1016 (1997).

32. Bossie, C. A. \& Sanders, M. M. A cDNA from Drosophila melanogaster encodes a lamin C-like intermediate filament protein. J. Cell Sci. 104, 1263 LP-1272 (1993).

33. Friedl, P., Wolf, K. \& Lammerding, J. Nuclear mechanics during cell migration. Curr. Opin. Cell Biol. 23, 55-64 (2011).

34. Zwerger, M. et al. Myopathic lamin mutations impair nuclear stability in cells and tissue and disrupt nucleo-cytoskeletal coupling. Hum. Mol. Genet. 22, 2335-2349 (2013).

35. Coffinier, C. et al. Deficiencies in lamin B1 and lamin B2 cause neurodevelopmental defects and distinct nuclear shape abnormalities in neurons. Mol. Biol. Cell 22, 4683-93 (2011).

36. Vergnes, L., Péterfy, M., Bergo, M. O., Young, S. G. \& Reue, K. Lamin B1 is required for mouse development and nuclear integrity. Proc. Natl. Acad. Sci. U. S. A. 101, 10428-33 (2004).

37. De vos, W. H. et al. Repetitive disruptions of the nuclear envelope invoke temporary loss of cellular compartmentalization in laminopathies. Hum. Mol. Genet. 20, 4175-4186 (2011).

38. Denais, C. M. et al. Nuclear envelope rupture and repair during cancer cell migration. Science (80-. ). 352, 353-358 (2016).

39. Raab, M. et al. ESCRT III repairs nuclear envelope ruptures during cell migration to limit DNA damage and cell death. Science (80-. ). 352, 359-362 (2016).

40. Irianto, J. et al. DNA Damage Follows Repair Factor Depletion and Portends Genome Variation in Cancer Cells after Pore Migration. Curr. Biol. 27, 210-223 (2017).

41. Dialynas, G. et al. LMNA variants cause cytoplasmic distribution of nuclear pore proteins in Drosophila and human muscle. Hum. Mol. Genet. 21, 1544-1556 (2012).

42. Ewen-Campen, B. et al. Optimized strategy for in vivo Cas9activation in \&lt;em\&gt;Drosophila\&lt;/em\&gt; Proc. Natl. Acad. Sci. 114, 9409 LP-9414 (2017). 